\title{
Les effets de la crise économique et financière sur les questions constitutionnelles en Écosse
}

The Impact of the Economic and Financial Crisis on Constitutional Issues in Scotland

\section{Edwige Camp-Pietrain}

\section{OpenEdition Journals}

Édition électronique

URL : http://journals.openedition.org/rfcb/959

DOI : $10.4000 /$ rfcb.959

ISSN : 2429-4373

Éditeur

CRECIB - Centre de recherche et d'études en civilisation britannique

\section{Référence électronique}

Edwige Camp-Pietrain, « Les effets de la crise économique et financière sur les questions

constitutionnelles en Écosse », Revue Française de Civilisation Britannique [En ligne], XXI-2 | 2016, mis en ligne le 10 septembre 2016, consulté le 20 avril 2019. URL : http://journals.openedition.org/ rfcb/959; DOI : 10.4000/rfcb.959

Ce document a été généré automatiquement le 20 avril 2019.

\section{cc)}

Revue française de civilisation britannique est mis à disposition selon les termes de la licence Creative Commons Attribution - Pas d'Utilisation Commerciale - Pas de Modification 4.0 International. 


\section{Les effets de la crise économique et financière sur les questions constitutionnelles en Écosse}

The Impact of the Economic and Financial Crisis on Constitutional Issues in Scotland

Edwige Camp-Pietrain

\section{Introduction}

1 En Écosse, les questions économiques et sociales sont étroitement liées aux questions constitutionnelles. En effet, le Parti travailliste, à l'origine de la dévolution du pouvoir, la concevait comme un moyen d'atteindre des finalités économiques et sociales, afin d'appliquer des politiques sociales-démocrates distinguant l'écosse du néo-libéralisme de Margaret Thatcher et de John Major. De même, le Scottish National Party (SNP) préconise l'accession de l'Écosse à l'indépendance et lui assigne des objectifs semblables, ainsi que la possibilité d'effectuer ses propres choix.

2 La crise économique et financière de 2008 a accentué les liens entre les questions économiques et sociales en Écosse, pour deux raisons principales. D'une part, c'est le SNP qui était au pouvoir à Édimbourg, ayant formé son premier gouvernement en 2007. Ce dernier s'est montré très critique à l'égard de la gestion de la crise par le gouvernement de Gordon Brown, mais aussi et surtout par celui de David Cameron, dénonçant sans relâche les politiques d'austérité. D'autre part, à partir de 2011, fort d'une majorité absolue au Parlement écossais, le gouvernement écossais du SNP a obtenu de son homologue britannique l'autorisation de convoquer un référendum d'autodétermination, aux termes d'un accord signé en octobre 2012. Dès lors, pendant les deux années suivantes, les indépendantistes ont cherché à convaincre les Écossais de voter en faveur de l'indépendance, afin de générer la croissance nécessaire à la réduction des inégalités. À l'inverse, les unionistes (conservateurs, libéraux-démocrates et travaillistes) ont défendu 
le cadre protecteur que constituait le Royaume-Uni. Si le 18 septembre 2014 les Écossais ont finalement rejeté l'indépendance, le débat s'est poursuivi en vue des élections à la Chambre des communes du 7 mai 2015. Le SNP a appelé à voter pour ses candidats afin de permettre à l'Écosse de disposer d'une voix forte à Westminster, pour fustiger l'austérité infligée aux Britanniques. Il a ainsi conquis la quasi-totalité des bastions travaillistes. Ce succès montre que son message a été entendu et lui permet de revendiquer davantage de leviers d'actions économiques pour le Parlement écossais (Holyrood) dans le Scotland Bill en discussion.

3 Cette analyse distinguera trois étapes chronologiques, entre 2007 et juillet $2015^{1}$ afin de montrer comment les partis politiques écossais, notamment le SNP, utilisent, et même instrumentalisent, les questions économiques et sociales, en particulier la crise économique et financière.

\section{Le premier gouvernement SNP (2007-2011) face à la crise : entre quête de la compétence et dénonciation de « bouc-émissaires »}

Entre 2007 et 2011, le gouvernement SNP, minoritaire, a oscillé entre quête de la compétence (le parti exerçant le pouvoir pour la première fois) et dénonciation de boucémissaires (les responsables étant à rechercher du côté de «Londres » afin de légitimer l'accession à l'indépendance)

\section{Une crise financière qui n'entame pas l'optimisme}

5 La crise financière qui a éclaté pendant cette période n'a pas entamé la confiance du gouvernement écossais qui avait à sa tête Alex Salmond, homme politique expérimenté siégeant à Holyrood, mais aussi à la Chambre des communes depuis 1987. Salmond, économiste de formation, avait exercé dans le secteur public (Scottish Office) et dans le secteur privé (Royal Bank of Scotland : RBS). Il agissait en étroite collaboration avec John Swinney, son ministre des Finances (ancien assureur) et Jim Mather, ministre des Entreprises (ancien chef d'entreprise).

Or à la fin de l'année 2008, RBS s'est trouvée à court de liquidités, tout comme un autre fleuron de la banque écossaise, Bank of Scotland, intégrée au groupe HBoS depuis 2001. Les deux établissements étaient victimes de leurs investissements dans des crédits à risque et de leur croissance démesurée. Le gouvernement britannique de Brown est intervenu en prenant des participations majoritaires et en garantissant les dépôts, dans un domaine réservé aux institutions britanniques. Néanmoins, le gouvernement écossais a fustigé une décision imposée de l'extérieur, impliquant l'absorption d'une banque écossaise, HBoS, par un groupe britannique, Lloyds, pour aboutir à la quasinationalisation de deux établissements écossais historiques². Il a également protesté contre la reprise par Nationwide de la société de crédit immobilier Dunfermline Building Society, car celle-ci était l'interlocuteur privilégié des bailleurs sociaux écossais ${ }^{3}$.

Le First Minister a affirmé que le gouvernement d'une Écosse indépendante aurait pu activer son plan de sauvetage. Mais la somme requise, plus de 100 milliards de livres, représentait trois fois le budget du Parlement écossais à l'époque. Il a continué à louer les pays de l' «Arc de prospérité » (Irlande, Islande, Danemark, Norvège, Finlande) alors que 
les deux premiers traversaient de graves difficultés. Il a notamment salué la réactivité du gouvernement de la République d'Irlande. En outre, selon le First Minister écossais, cette crise résultait de la réglementation laxiste des gouvernements New Labour et des pratiques de quelques financiers (ventes à découvert) ${ }^{4}$. Mais il se refusait à mettre en cause les banques écossaises et leurs dirigeants. Il avait d'ailleurs désigné Sir George Mathewson, ancien PDG de RBS, à la tête de son groupe de conseillers économiques ${ }^{5}$. Or, Sir Mathewson, qui avait été l'artisan de la croissance de la banque, était à l'origine de la nomination de Fred Goodwin à sa direction générale. Lorsque la crise financière a éclaté, il dirigeait un fonds spéculatif.

\section{Une crise économique lointaine}

8 La crise économique paraissait lointaine. Grâce à la dotation britannique dont la progression était garantie par la formule Barnett, le gouvernement Salmond disposait de ressources lui permettant de mener des politiques présentées comme des emblèmes de la social-démocratie à l'écossaise, telles l'abrogation totale des droits universitaires et la suppression du ticket modérateur sur les médicaments. Pour faire adopter ces réformes, le SNP a dû s'assurer du soutien respectif des libéraux-démocrates et des travaillistes ${ }^{6}$. Or ces deux partis, qui avaient gouverné ensemble l'Écosse de 1999 à 2007, avaient appliqué politiques comparables (réduction substantielle des droits universitaires, prise en charge des frais de soins personnels des personnes âgées dépendantes). De même, le gouvernement de Salmond a maintenu le caractère public des services publics, qui avait été restauré par ses prédécesseurs, les écoles publiques ne pouvant s'affranchir de la tutelle des collectivités territoriales tandis que le National Health Service (NHS) n'avait quasiment pas recours à des sociétés privées.

Cependant, lors du seul vote décisif de chaque session parlementaire, relatif au projet de loi de finances, ce sont les députés conservateurs qui ont approuvé le budget du SNP, notamment grâce à sa politique fiscale ${ }^{7}$. Il avait en effet gelé la taxe locale sur les personnes (council tax) et abaissé la taxe locale sur les entreprises (business rates) pour les PME. Cette orientation, conçue en 2001, visait notamment à modifier la perception du projet indépendantiste par les milieux d'affaires. Swinney, alors leader du parti, ainsi que Salmond et Mather, avaient multiplié les réunions avec des petits groupes de chefs d'entreprises. Ainsi, le gouvernement SNP affichait sans complexe des politiques socialesdémocrates côté dépenses, et néo-libérales côté recettes. Mais la portée de ces annonces était relativement limitée. En effet, les ressources propres du gouvernement écossais étant très minces (outre les taxes locales, un pouvoir résiduel sur le seul taux de base de l'impôt sur le revenu, jamais utilisé depuis 1999), il n'avait pas à se préoccuper du financement de ses dépenses.

\section{La nécessité de responsabiliser le Parlement écossais}

10 C'est pour remédier à ce décalage que les trois partis britanniques (le Parti travailliste, alors au pouvoir sur le plan britannique, mais aussi les conservateurs et les libérauxdémocrates) ont élaboré un projet visant à accroître les pouvoirs fiscaux du Parlement écossais. Ce projet résultait de réflexions menées par une commission indépendante, dirigée par Kenneth Calman. Son rapport, publié en 2009, a servi de base au Scotland Bill, déposé par le gouvernement Cameron en novembre 2010 et adopté au printemps 2012, 
sous le nom de Scotland Act. Le gouvernement écossais, à l'écart de cette réflexion, a multiplié les critiques. D'une part, il estimait que les impôts confiés à Holyrood n'étaient pas suffisants pour mener des politiques ayant un réel impact économique. En effet, s'agissant de l'impôt sur le revenu, les trois taux britanniques seraient abaissés de 10 points de pourcentage en Écosse et il appartiendrait au Parlement écossais de voter un complément. Ce dernier aurait également la maitrise de quelques impôts mineurs (droit de timbre sur les mutations foncières à titre onéreux, taxe sur l'enfouissement des déchets non recyclés). Par contre, il ne pourrait gérer l'impôt sur les sociétés alors que le SNP voulait s'aligner sur le taux de la République d'Irlande (12,5\%), inférieur de moitié au taux alors en vigueur au Royaume-Uni. D'autre part, le gouvernement écossais critiquait les contraintes inhérentes à ces transferts. Ainsi, le complément écossais à l'impôt sur le revenu, identique pour chaque taux d'imposition, ne serait pas progressif. Quant au nouveau pouvoir d'emprunt pour dépenses d'investissement, il était trop restrictif.

11 À l'issue des élections au Parlement écossais de 2011, grâce à sa majorité absolue de sièges, le gouvernement écossais semblait plus représentatif de la population locale. Il a obtenu du gouvernement britannique quelques amendements au projet, notamment au sujet de la révision des modalités de la baisse de la dotation écossaise, pour tenir compte des nouvelles ressources propres. Ainsi, les députés SNP ont finalement approuvé le Scotland Bill de 2012, à Holyrood à titre consultatif et à la Chambre des communes à titre formel ${ }^{8}$. Ils n'ont pas pour autant renoncé à mettre en évidence les limites de ce texte au regard de leur programme d'autonomie fiscale complète, conçu en 2001, pour permettre au Parlement écossais de gérer tous les impôts, en versant une contribution au budget britannique pour financer les domaines réservés résiduels comme la défense, la politique étrangère et la monnaie. Le gouvernement écossais laissait entendre qu'il pourrait soumettre ce projet à l'électorat, aux côtés de l'accession à l'indépendance, dans le référendum d'autodétermination qui constituait l'objectif du SNP9'.

\section{L'indépendance, un moyen de créer une société prospère et équitable?}

12 Fort de sa majorité absolue, le gouvernement de Salmond a pu négocier avec Cameron l'organisation d'un référendum portant exclusivement sur l'indépendance. Pendant la campagne, les thèmes économiques et sociaux étaient au cœur des débats.

\section{Les finalités sociales de l'indépendance}

13 Pour les indépendantistes, réunis au sein de Yes Scotland, l'indépendance de l'Écosse devait permettre de rompre avec les politiques d'austérité du gouvernement Cameron. Le gouvernement SNP, soucieux de crédibilité, a nommé plusieurs commissions de réflexion; l'une d'entre elles, qui émanait de son conseil économique (et comptait deux lauréats du Prix Nobel) était chargée des questions fiscales ; une seconde s'est intéressée à la protection sociale.

14 S'agissant des dépenses, le SNP promettait le retour à l'État Providence des origines, avec des services publics gratuits de qualité, gérés par les autorités publiques. Les prestations sociales seraient fondées sur les besoins et non sur des contraintes, des évaluations et des plafonnements. Le gouvernement SNP estimait être en mesure de maintenir le niveau de 
toutes les allocations, voire même de l'améliorer en tenant compte des conditions écossaises, l'espérance de vie plus courte qu'en Angleterre pouvant justifier un départ à la retraite anticipé ${ }^{10}$.

En ce qui concerne les recettes, le SNP voulait tout d'abord optimiser les ressources naturelles de l'Écosse, en particulier les hydrocarbures en mer du Nord, piliers de son programme économique depuis leur découverte au début des années soixante-dix. Le SNP ne cessait de citer le pic de recettes en 2008-2009 (12 milliards de livres). $78 \%$ de cette somme devait revenir à l'Écosse au regard du droit international, ce qui représenterait près de $20 \%$ de son budget. Le SNP reconnaissait le caractère fluctuant de ces recettes (entre 2 et 12 milliards annuels, en livres courantes, depuis le début des années quatrevingt), ainsi que le déclin de la production. C'est la raison pour laquelle il envisageait des incitations fiscales, tant pour le démantèlement des puits anciens que pour la recherche et l'exploration de nouveaux gisements. Mais il continuait à promettre l'instauration d'un fonds abondé par une partie des recettes, sur le modèle de la Norvège. De plus, le SNP misait sur les énergies renouvelables, affirmant que grâce à ses façades maritimes, l'Écosse disposait de $10 \%$ à $25 \%$ du potentiel de l'UE. Ensuite, le gouvernement SNP entendait stimuler la croissance économique. Il voulait attirer les entreprises en abaissant l'impôt sur les sociétés et en évitant d'alourdir les contraintes réglementaires ${ }^{11}$. Il cherchait également à séduire la main-d'œuvre par une politique d'immigration attractive. Enfin, le SNP escomptait des économies, grâce à une gestion rigoureuse des finances publiques et à l'abandon du nucléaire sous toutes ses formes, civiles et militaires.

L'équilibre entre recettes et dépenses ne serait pas assuré au regard des statistiques officielles pour 2013-2014. L'Écosse connaîtrait un déficit qui, rapporté au PIB, serait comparable au déficit britannique (5,9\% contre $5,8 \%$ ): il serait donc viable ${ }^{12}$. De plus, non seulement ce déficit était resté en deçà du déficit britannique depuis l'éclatement de la crise financière mais, qui plus est, il n'était pas inéluctable car jusqu'en 2008-2009, l'Écosse enregistrait des excédents. Les nationalistes soulignaient également que les revenus fiscaux collectés en Écosse (9,1\% des recettes britanniques en 2012-2013) dépassaient la part de celle-ci dans la population britannique (8,3\%). S'ils étaient alors inférieurs à la part des dépenses publiques revenant à l'Écosse (9,3\%), ils avaient toujours été supérieurs à celle-ci au cours des cinq années précédentes.

Le SNP était donc relativement confiant quant aux capacités de l'Écosse ${ }^{13}$. Dans le cadre de la dévolution, elle avait rattrapé une partie de son retard, se situant désormais derrière Londres et le Sud-Est pour le taux de chômage ou la richesse produite. Elle obtenait même des résultats meilleurs que la moyenne britannique pour le taux d'activité ou le coefficient de Gini (mesurant les inégalités) ${ }^{14}$. L'indépendance lui conférerait des possibilités décuplées, grâce aux atouts liés à sa petite taille ; or entre les années soixante et 2007, les petits États européens avaient toujours affiché un taux de croissance supérieur à celui du Royaume-Uni. Cette richesse permettrait de réduire les inégalités sociales, elles-mêmes présentées comme autant de freins à la croissance. Au final, grâce à la hausse de la population, de l'emploi et de la productivité, les recettes fiscales progresseraient de cinq milliards d'ici 2029-2030, ce qui représenterait 1000 livres supplémentaires par foyer.

18 Si cet optimisme faisait l'unanimité au sein du camp indépendantiste, nombre d'alliés du SNP avaient une vision sensiblement différente d'une Écosse indépendante. Il fallait, selon eux, créer une autre société. Ainsi, les verts, le Scottish Socialist Party, la Radical Independence Campaign, et certains membres du SNP, hostiles aux cadeaux aux entreprises, 
prônaient une hausse de la pression fiscale sur les bénéfices et sur les hauts revenus. Ils appelaient à un renforcement de la réglementation du travail dans un sens favorable aux salariés, par exemple l'abolition des contrats de travail sans heures garanties. Ils ne considéraient pas la croissance économique comme la seule mesure du progrès. Certains d'entre eux mettaient même en cause la recherche de nouveaux puits de pétrole.

\section{L'indépendance comme source d'appauvrissement} données. Ils soulignaient la volatilité des recettes pétrolières (passées de 10,9 à 6,2 milliards entre 2011-2012 et 2012-2013) qui contraindrait la construction d'un budget. De plus, ces recettes connaissaient une régression, plus accentuée en livres constantes qu'en livres courantes : le pic de 2008-2009 était ainsi largement inférieur à celui de 1985. Les unionistes mettaient en avant le décalage entre les dépenses publiques et les revenus. Ils contestaient les pourcentages du SNP (comparaisons entre des dépenses et des recettes exprimées en proportion de celles du Royaume-Uni), préférant les chiffres bruts, rapportés au nombre d'habitants. Ils montraient ainsi que l'écart entre dépenses et revenus était plus marqué pour l'Écosse, même avec les ressources pétrolières (10 100 contre 12800 livres en 2012-2013), que pour l'ensemble du Royaume-Uni (9 400 contre 11 300). Or les baisses d'impôts promises par le SNP réduiraient les rentrées dans un premier temps, tandis que les coûts inhérents à la création d'un État alourdiraient les dépenses. Les unionistes insistaient également sur le creusement de l'écart entre les déficits écossais et britannique au cours des dernières années.

Les unionistes affirmaient que l'arc de prospérité s'était transformé en " arc de faillite ». Ces États avaient dû consentir des efforts budgétaires considérables pour sauver leur secteur bancaire. L'Écosse se serait trouvée dans une situation comparable en 2008, avec des actifs financiers représentant plus de $1254 \%$ de son PIB (contre $492 \%$ pour le Royaume-Uni). De ce fait, les unionistes ont également remis en cause la possibilité, pour une Écosse indépendante, de conserver la livre dans le cadre d'une union monétaire avec le reste du Royaume-Uni. Cette perspective était jugée trop risquée pour le contribuable britannique selon une analyse du Trésor publiée en février 2014, aussitôt reprise par George Osborne, le Chancelier de l'Échiquier ${ }^{15}$, et ses alliés.

Par conséquent, l'Écosse serait viable sur le court-terme; mais elle s'appauvrirait par rapport au reste du Royaume-Uni. Sa dette ne cesserait de croître et elle n'aurait plus les moyens de définir des politiques autonomes. Elle serait contrainte de pratiquer des politiques d'austérité plus sévères que celles du gouvernement britannique, alors qu'elle subirait un surcoût de plus d'un point sur les taux d'intérêt de ses emprunts. Ainsi, le gouvernement britannique a estimé que chaque habitant devrait acquitter 1400 livres supplémentaires dans une Écosse indépendante, en tenant compte de l'écart entre ses recettes et ses dépenses, des coûts de création d'un État, du financement des emprunts et des politiques promises par les nationalistes ${ }^{16}$.

Les unionistes préféraient transférer des pouvoirs complémentaires au Parlement écossais, afin de continuer à bénéficier de la mutualisation des ressources. Les libérauxdémocrates avaient déjà approfondi leur réflexion, alors que leurs partenaires, en proie à des divisions internes, n'ont finalisé leur projet qu'au printemps $2014^{17}$. Leurs leaders écossais se sont engagés à les mettre en œuvre. Les leaders britanniques en ont fait le 
« serment » à l'approche du scrutin, lorsque l'indépendance semblait pouvoir rassembler une majorité.

Qui plus est, les unionistes encourageaient le gouvernement écossais à faire usage de ses compétences. Les travaillistes, qui avaient des objectifs socio-économiques comparables à ceux du SNP, pensaient pouvoir les atteindre dans le cadre de la dévolution. Au début de 2014, ils ont ainsi contraint le gouvernement écossais à apporter une compensation intégrale aux Écossais concernés par la bedroom tax, cette réduction de l'allocationlogement pour les locataires d'un logement social en cas de chambre inoccupée. Mais les syndicats, alliés traditionnels des travaillistes, étaient plus mitigés; alors que leur confédération Scottish Trades Union Congress (STUC) avait été le moteur de l'avènement d'un Parlement écossais, elle n'a pas pris parti dans la campagne référendaire ${ }^{18}$.

\section{Une réponse ambiguë de l'électorat}

Le 18 septembre 2014, 55 \% des Écossais ont rejeté l'indépendance. Mais les électeurs unionistes, comme les électeurs indépendantistes, étaient motivés par des considérations économiques et sociales ${ }^{19} .57 \%$ des personnes ayant coché le «non » sur leur bulletin de vote évoquaient la monnaie, 37 \% les impôts et les dépenses publiques. Les craintes des partis unionistes, qui ont redoublé à l'approche du scrutin, avec le soutien de grands établissements bancaires, ont été entendues par les derniers indécis. Quant aux partisans de l'indépendance, si la priorité de $74 \%$ d'entre eux était la rupture avec le système politique britannique, plus d'un tiers citait les impôts et les dépenses publiques, ainsi que la défense du NHS. Le SNP avait en effet martelé, tout au long du mois d'août, que ce dernier était en voie de privatisation en Angleterre, ce qui ne manquerait pas d'avoir des effets délétères en Écosse. Les discours offensifs de Salmond, opposant l'explosion des banques alimentaires au renouvellement de Trident, ont marqué les esprits.

Le vote en faveur de l'indépendance a été privilégié par les personnes modestes ${ }^{20}$ : les classes ouvrières (53,6 \%, contre $41,7 \%$ des classes moyennes), les personnes ayant les revenus les plus bas $(56,4 \%$ pour le premier quartile, contre $46,8 \%$ pour le dernier quartile), les locataires $(61,9 \%$, contre $35,4 \%$ des propriétaires-occupants). À ces déterminants objectifs s'ajoutait le positionnement des individus sur l'échiquier politique. Ainsi, les classes moyennes se situant à gauche étaient plus susceptibles de voter « oui » que les classes ouvrières dans leur ensemble ${ }^{21}$.

Par ailleurs, les votes étaient comptabilisés dans les 32 collectivités territoriales. Les habitants de quatre d'entre elles se sont prononcés pour l'indépendance. Ce sont d'anciens centres industriels, qui, tel Glasgow, comptent de nombreux quartiers défavorisés, parmi les plus pauvres du Royaume-Uni. Leurs habitants, qui ont retrouvé une occasion de croire en la chose publique, se sont déplacés en masse pour prendre part au scrutin ${ }^{22}$ et plébisciter l'indépendance. Ainsi, on peut observer une corrélation non négligeable entre la proportion de vote « oui » et le taux de chômage dans les collectivités territoriales ${ }^{23}$. Cet espoir ne s'est pas éteint le 19 septembre.

Le SNP comme rempart contre l'austérité lors des élections à la Chambre des communes de 2015 Les politiques économiques et sociales, à travers le prisme de la question institutionnelle, ont a continué à occuper le devant de la scène. 


\section{De nouvelles compétences pour le Parlement écossais avec des finalités socio-économiques}

$\mathrm{Au}$ lendemain du référendum, les partis unionistes ont tenu leur engagement. David Cameron a désigné une commission de réflexion, présidée par Lord Smith of Kelvin, afin d'élaborer un projet de transfert de pouvoirs au Parlement écossais. Les Verts et le SNP ont participé à ces travaux.

Les recommandations de cette commission, publiées à la fin du mois de novembre $2014^{24}$, doivent permettre au Parlement écossais de prendre des dispositions attendues pendant la campagne référendaire. Ainsi, il aura la maîtrise d'impôts correspondant à plus de $50 \%$ de ses dépenses: taux et tranches de l'impôt sur le revenu, taxes aéroportuaires, redevances du Crown estates (propriétés de la Couronne, notamment sur le littoral écossais). Il recevra la moitié du produit de la TVA collectée en Écosse. Il se verra confier, pour la première fois, des allocations (adultes handicapés), ainsi que la possibilité d'en créer et d'abonder les prestations britanniques. Holyrood pourra abolir la bedroom tax et modifier la périodicité des versements de l'universal credit, tant décrié. En matière de droit du travail, les Écossais pourront gérer le programme de retour à l'emploi, les indemnités en cas d'accidents de travail, et les tribunaux de prud'hommes (ce qui leur permettra de supprimer les droits à acquitter pour déposer une plainte). Quant à l'énergie, le Parlement écossais aura la maitrise des licences d'exploitation des gaz de schiste, il pourra orienter le secteur des énergies renouvelables (par l'intermédiaire du Crown estates ) et lutter contre la précarité énergétique en imposant des contraintes aux sociétés de distribution.

Cependant, dans cette période pré-électorale, le consensus s'est fissuré dès janvier 2015 lorsque le gouvernement Cameron a publié un avant-projet fondé sur ces recommandations $\mathrm{s}^{25}$. Les travaillistes ont réclamé la dévolution complète de l'allocationlogement et la dévolution anticipée du programme de retour à l'emploi. Le SNP a estimé que l'avant-projet trahissait le rapport Smith à cause des possibilités de veto du gouvernement britannique.

\section{Le programme du SNP au cœur de la campagne électorale}

31 Le SNP souhaitait aller au-delà du rapport qu'il avait signé. Il a repris son projet d'autonomie fiscale, désormais qualifiée de "responsabilité » fiscale complète, afin de démontrer sa capacité à équilibrer les comptes.

De ce fait, les partis unionistes ont dénoncé les risques pour l'Écosse. Les statistiques pour 2013-2014, lorsque le cours du pétrole était encore élevé, montraient une progression de l'écart entre les déficits écossais et britannique $\left(6,4 \%\right.$ contre $4,1 \%$ du $\left.\mathrm{PIB}^{26}\right)$. Les projections de l'Institute for Fiscal Studies, organisme indépendant, prévoyaient un creusement du déficit écossais, suivi d'une réduction, lente, en raison de l'effondrement du baril au second semestre 2014 ${ }^{27}$. Le déficit britannique diminuerait plus rapidement, avant de disparaitre en 2018-2019. La polémique s'est focalisée sur le décalage entre les déficits écossais et britannique en 2015-2016, lequel correspondrait à 7,6 milliards de livres $^{28}$. Pour le combler, le gouvernement écossais devrait augmenter les impôts et/ou abaisser les dépenses publiques, et ce, dans des proportions difficilement supportables par la population. 
33 Nicola Sturgeon, qui avait succédé à Salmond à la tête du SNP et du gouvernement écossais, a estimé que ces prévisions ne tenaient pas compte de tous les paramètres. Les sondages laissant penser que le SNP pourrait emporter les élections en Écosse et fournir une force d'appoint au Parti travailliste à Westminster, elle a abandonné la promesse de réduction générale de l'impôt sur les sociétés, lui préférant des baisses ciblées ${ }^{29}$. Elle a approuvé les hausses d'impôts sur les personnes les plus aisées et sur les banques, prévues par le manifeste électoral travailliste ${ }^{30}$. Côté dépenses, elle a maintenu un objectif de réduction des inégalités, en multipliant les symboles, parmi lesquels des nominations : entrée dans son conseil économique de Harry Burns (ancien directeur des services de santé, connu pour ses réflexions sur les inégalités), création d'un contrôleur indépendant de l'impact de ses politiques sur la pauvreté (mission confiée à Naomi Eisenstadt, fort impliquée dans la lutte contre la pauvreté des enfants) ${ }^{31}$. De même, Sturgeon a fait voter une loi éteignant tout reliquat de dettes pour non-paiement de la poll tax, réforme de Thatcher tellement honnie en Écosse que son souvenir était encore vivace ${ }^{32}$. Elle a créé une Convention sur le travail équitable, composée de représentants des partenaires sociaux, afin de rédiger un cadre de référence d'ici le printemps 2016. Mais la First Minister était également désireuse de diversifier les solutions, en matière de conditions de travail ou de gestion des services publics (ce qu'elle a signifié en visitant une école à Londres). Étant elle-même issue de la classe ouvrière, elle était plus crédible pour porter ce message que son prédécesseur. De plus, au moment où Cameron cherchait à attiser les craintes des électeurs anglais à l'égard d'une alliance entre travaillistes et SNP, la First Minister s'est présentée, lors des débats télévisés, comme un rempart contre l'austérité. Elle apportait une solution distincte du discours dominant, en affichant l'intention de mettre fin aux déficits de façon progressive.

La réponse à la chute des cours pétroliers a également alimenté la polémique. Certes, en mars 2015 , le gouvernement britannique a supprimé la quasi-totalité de la hausse de la surtaxe infligée aux sociétés pétrolières en 2011 tout en accordant des abattements par bassin, afin d'encourager l'investissement dans les puits les plus difficiles d'accès. Fergus Ewing, ministre écossais de l'Énergie, jugeait toutefois cela insuffisant, nombre de puits n'étant plus rentables avec un baril à 60 dollars $^{33}$. Le gouvernement écossais était luimême pressé d'agir par l'opposition en Écosse. S'il a instauré un groupe de travail, il s'est refusé à envisager le «fonds de résilience " prôné par les travaillistes, car ce fonds, destiné aux collectivités territoriales affectées par les licenciements dans l'industrie pétrolière, aurait été alimenté par des prélèvements sur les bénéfices de celle-ci ${ }^{34}$.

\section{Un résultat susceptible de renforcer l'articulation entre questions socio-économiques et institutionnelles}

La stratégie de Sturgeon a porté ses fruits. Le SNP a emporté 56 des 59 sièges écossais à la Chambre des communes, avec $50 \%$ des suffrages, en moyenne et dans plus de la moitié des circonscriptions, mettant un terme à 50 ans de domination travailliste. Il a recueilli les suffrages des électeurs ayant opté pour l'indépendance, notamment les anciens électeurs travaillistes, en endossant le programme du Parti travailliste alors que l'image de ce dernier avait été écornée par son association avec les Conservateurs, symboles de l'austérité, pour sauver l'Union en 2014. Ainsi, une corrélation forte est apparue entre le vote SNP et le taux de chômage, avec un coefficient de 0,6, comparable à celui du Parti travailliste, ce dernier étant en déclin par rapport au scrutin de $2010^{35}$. 

publication du Scotland Bill par le gouvernement Cameron, sur la base de l'avant-projet, les nationalistes se sont érigés en défenseurs du rapport Smith, avec le soutien de la commission multipartite de Holyrood qui venait de publier un rapport critiquant les restrictions dans la définition des domaines dévolus. Ils souhaitaient même aller au-delà car en l'état, Holyrood ne disposerait que $29 \%$ des revenus fiscaux et $36 \%$ des dépenses publiques en Écosse ${ }^{36}$. Ils réclament le transfert de leviers d'action économique. Grâce à la dévolution de pans entiers du droit du travail (salaire minimum, lois syndicales, hygiène et santé), les institutions écossaises pourraient améliorer les conditions de travail des salariés et de leurs défenseurs, par exemple en généralisant un niveau de salaire minimum permettant de subvenir aux besoins élémentaires (living wage). La dévolution de la politique d'égalité des chances faciliterait la lutte contre les discriminations. La gestion des prélèvements sur les entreprises (impôt sur les sociétés, cotisations sociales, taxe sur les plus-values) permettrait d'accorder des baisses ciblées sur les investissements et la recherche-développement. En matière de cotisations, le SNP entend maîtriser d'autres composantes de l'universal credit, ainsi que les règles d'attribution et de sanctions, afin de pratiquer des revalorisations en faveur de publics prioritaires. Quant à l'autonomie fiscale complète, il s'agit désormais d'un objectif sur le moyen terme, mais le gouvernement écossais souhaiterait en jouir au moment qu'il jugera opportun, tout en continuant à tirer avantage de la formule Barnett dans la période transitoire ${ }^{37}$.

Nombre de ces exigences ont pour objectif d'embarrasser les travaillistes plongés dans une remise en cause risquant de les précipiter vers une autre déroute lors des élections à Holyrood de 2016. Les travaillistes défendent en effet l'uniformité des droits et de devoirs des salariés britanniques. Or le STUC soutient le gouvernement SNP dans sa quête de leviers économiques, l'objectif de réduction des inégalités légitimant certaines divergences ne remettant pas en cause la cohésion britannique. Mais il ne cautionne pas l'autonomie fiscale complète, par crainte de dumping fiscal entre les nations ${ }^{38}$. Quant à la protection sociale, le mouvement travailliste (parti et syndicats) demeure attaché à ce pilier de l'unité du Royaume-Uni.

Les travaillistes soulignent que si le Parlement écossais était maître de sa fiscalité, le déficit deviendrait abyssal car au milieu de l'année 2015, les prévisions de recettes pétrolières sont de plus en plus pessimistes, sous le double effet de l'effondrement des cours mais aussi de la production ${ }^{39}$. Ils s'interrogent sur les leviers économiques prônés par le SNP. Ainsi, s'agissant du living wage, le gouvernement écossais en fait bénéficier ses salariés, tout en se contentant d'inciter les entreprises privées à faire de même, alors qu'il pourrait être plus exigeant dans ses appels d'offre ${ }^{40}$. En ce qui concerne le programme de retour à l'emploi, ils voudraient contraindre le gouvernement écossais à en confier la gestion aux collectivités territoriales, afin de l'adapter aux marchés locaux du travail ${ }^{41}$.

Plus généralement, les travaillistes, comme les deux autres partis unionistes, exhortent le gouvernement SNP à faire usage des compétences qu'il détient au lieu d'en réclamer sans cesse de nouvelles. S'agissant des pouvoirs fiscaux, il va devoir prendre position dès 2016, avec la dévolution partielle de l'impôt sur le revenu prévue par le Scotland Act de 2012. Or, le SNP ne peut ignorer l'environnement de l'Écosse. Ainsi, lors de l'entrée en vigueur de la nouvelle taxe sur les mutations foncières à titre onéreux le $1^{\mathrm{er}}$ avril 2015, Swinney, toujours ministre des Finances, a augmenté la taxation des propriétés les plus luxueuses, afin d'améliorer la progressivitéét ${ }^{42}$ Mais il a dû s'adapter au barème adopté par Osborne en Angleterre, en abaissant la taxe sur les transactions les plus modestes. Qui plus est, le

Revue Française de Civilisation Britannique, XXI-2 | 2016 
gouvernement écossais devra s'interroger sur la cohérence de sa politique fiscale. Au début de 2015, il a instauré une commission de réflexion consacrée à la fiscalité locale ; les conservateurs s'en sont désolidarisés, car ils envisagent de se démarquer en prônant une baisse des impôts. Le SNP, tenté par ce principe, se garde pourtant de prendre ce type d'engagement. Par ailleurs, les ministres du gouvernement écossais anticipent des conflits avec leurs homologues britanniques car nombre de domaines seront partagés (s'agissant de l'impôt sur le revenu, le gouvernement britannique continuera à définir les revenus et les abattements). Ils soulignent la difficulté à exercer un impact réel sur l'économie par l'intermédiaire d'un nombre réduit d'impôts. Ils affirment même que toute stimulation de l'activité économique écossaise profitera à Westminster grâce à l'impôt sur les sociétés notamment.

Quant aux dépenses, le SNP ne cesse de mettre en avant ses politiques emblématiques, ce qui, selon l'opposition, permet d'occulter les coupes opérées par ailleurs. Ainsi, si les étudiants écossais n'acquittent plus de droits universitaires, ils reçoivent des bourses inférieures à celles des Anglais ${ }^{43}$. Les collectivités territoriales n'ayant plus les moyens d'appliquer les politiques du gouvernement écossais, sous le double effet de la baisse de leur dotation et du gel de la fiscalité locale, les personnes âgées dépendantes se voient réclamer des frais pour leurs soins personnels ${ }^{44}$. Si le NHS conserve un caractère public en Écosse, le budget qui lui est consacré n'a pas été maintenu, à la différence de l'Angleterre, ce qui se traduit par une pénurie de personnels et un engorgement des services d'urgence ${ }^{45}$. Les unionistes dénoncent également le manque de logements sociaux et la réduction des places disponibles dans l'enseignement professionnel, malgré les annonces officielles. Or, le gouvernement de Sturgeon a continué à rendre "Londres » responsable de ces difficultés, tant lors des coupes budgétaires annoncées peu après les élections législatives qu'à l'issue du rejet de tous les amendements du SNP au Scotland Bill.

\section{Conclusion}

41 Le débat public écossais est monopolisé par les questions socio-économiques à travers les questions institutionnelles. La poursuite de la dévolution, comme l'accession à l'indépendance, sont présentées comme des moyens de stimuler la croissance et de réduire les inégalités. En réalité, ce sont les considérations économiques qui priment puisque la persistance d'inégalités est décrite comme un frein à la croissance.

Le programme du SNP a séduit près de la moitié de l'électorat écossais, lors des élections de 2011 au Parlement écossais, puis lors du référendum de 2014 et lors des élections de 2015 à la Chambre des communes. Il correspondait à leurs aspirations et se situait en rupture avec « Londres ». Mais ces électeurs ne semblaient pas se préoccuper du décalage entre la rhétorique du SNP, efficace pour gagner les élections, et son bilan au gouvernement écossais depuis 2007, moins flamboyant. Celui-ci a évité les engagements en matière fiscale, tout en brandissant des politiques sociales emblématiques masquant des situations contrastées. En pratique, le gouvernement SNP se démarque de ses modèles du Nord de l'Europe dans lesquels la fiscalité, lourde, est redistributive. Les Écossais ne semblent guère plus disposés que les Anglais à accepter ce type d'efforts.

43 Au sein du Royaume-Uni, le gouvernement SNP entend se distinguer. Mais il consacre plus de temps à réclamer des pouvoirs qu'à exercer ceux qu'il détient. De plus, il accuse le gouvernement britannique de toute réduction budgétaire l'empêchant d'agir, notamment pour contenir la pauvreté. Néanmoins, certaines décisions du gouvernement Cameron 
sont proches de son programme. Ainsi, en juin-juillet 2015, celui-ci a annoncé une baisse de l'impôt sur les sociétés (au niveau prôné par le SNP) ainsi que la mise en place d'un " living wage » (7,20 livres l'heure, alors que le gouvernement écossais retient le taux de 7,85 livres). En outre, le gouvernement Sturgeon n'a pas le monopole des griefs à l'encontre du pouvoir central. La campagne référendaire a libéré la parole au pays de Galles et en Irlande du Nord, où les assemblées locales revendiquent des pouvoirs fiscaux. Elles devraient obtenir satisfaction respectivement pour une partie de l'impôt sur le revenu et pour l'impôt sur les sociétés. Or, elles n'ont pas l'intention d'accroitre la pression fiscale. De plus, leurs dirigeants, qui estiment que la formule Barnett avantage l'Écosse (alors que celle-ci a rattrapé son retard sur le reste du Royaume-Uni), veulent la remplacer par une grille fondée sur les besoins de chaque nation.

La position du gouvernement britannique n'est pas non plus dépourvue d'ambiguïtés. Le Premier ministre a plusieurs fois décrit le Royaume-Uni comme une famille, dont les composantes devaient être respectées. Dans le même temps, dès le lendemain du référendum, il a promis de restreindre l'influence des députés écossais à la Chambre des communes sur les textes concernant l'Angleterre, notamment sur le vote de l'impôt sur le revenu. Or, la rupture de ce socle commun ouvrirait une brèche dans l'unité du royaume, comme le clament les travaillistes. De plus, nombre de textes anglais ayant des répercussions sur le budget écossais par la formule Barnett, les Écossais seraient privés d'un droit de regard sur des questions affectant leurs administrés ${ }^{46}$. En outre, le gouvernement Cameron semble répondre aux requêtes des assemblées locales au cas par cas. Il refuse de convoquer la Convention constitutionnelle réclamée par les travaillistes pour donner de la cohérence aux réformes. Enfin, Cameron est déterminé à poursuivre ses politiques d'austérité, ce qui va permettre au SNP d'attiser le ressentiment des Écossais, notamment lors de l'entrée en vigueur des nouvelles compétences de Holyrood, car elles reposent sur une coopération étroite entre les deux gouvernements. Cela pourrait, comme l'a laissé entendre dès le mois de juin 2015 Stewart Hosie, numéro deux du parti, justifier un second référendum d'autodétermination.

Edwige Camp-Pietrain est professeur des Universités. Elle enseigne la civilisation britannique à l'Université de Valenciennes. Ses recherches portent sur la vie politique, les institutions et les politiques publiques dans l'Écosse contemporaine.

\section{BIBLIOGRAPHIE}

CAMP-PIETRAIN Edwige, L'Impossible indépendance écossaise?, Neuilly, Atlande, octobre 2014.

CBI SCOTLAND, Submission to the Smith Commission, Glasgow, octobre 2014.

COMMISSION ON THE SCOTTISH CONSTITUTION, Serving Scotland Better, Édimbourg, 2009.

EXPERT GROUP ON WELFARE, Rethinking Welfare: Fair, Personal and Simple, Édimbourg, Scottish

Government, mai 2014. 
FINDLAY Neil, KANE Tommy \& LEONARD Richard, « Powers for Radical Change », in BRYAN Pauline (dir.), Scotland. Myths, Realities and Radical Future, Glasgow, Red paper collective, 2015, pp. 11-14. FISCAL COMMISSION WORKING GROUP, Principles for a Modern and Efficient Tax System in an Independent Scotland, Édimbourg, Scottish Government, novembre 2013.

HENDERSON Ailsa \& MITCHELL James, The Scottish Question, Six Months On, Édimbourg, University of Edinburgh, 27 mars 2015.

HM GOVERnMent (HMG), Scotland Analysis: Assessment of a Sterling Currency Union, Londres, Stationery Office, $\mathrm{Cm}$ 8815, février 2014.

HMG, Scotland Analysis: Fiscal Policy and Sustainability, Londres, Stationery Office, Cm 8854, mai 2014.

HOUSE OF COMMONS (HC), Hansard, 26 avril 2012, col. 1133-1179.

HC, Hansard, 8 juin 2015, col. 913-1013.

HC, Hansard, 15 juin 2015, col. 24-153.

HC, Hansard, 30 juin 2015, col. 1339-1450.

HC, Hansard, 15 juillet 2015, col. 936-1050.

HC, Scotland Act 2012, Londres, Stationery Office, c. 11.

HC, Scotland Bill, Londres, Stationery Office, 56/1.

MACWHIRTER Iain, Disunited Kingdom. How Westminster Won a Referendum but Lost Scotland, Glasgow, Cargo, 2014.

MCLEAN Christopher \& THOMSON Douglas, « Characterisation of the Disadvantaged: Explaining Differences in Levels of Support for Independence by Income Levels, Economic Activity and Socio-Economic Status », Scottish Affairs, vol. 23, n¹, 2014, pp. 125-147.

N-56, Oil and Gas. A Long-Term Plan to Maximise Economic Impact, Édimbourg, mars 2015.

PATERSON Lindsay, « Utopian Pragmatism: Scotland's Choice », Scottish Affairs, vol. 24, n 1,2015 , pp. 22-46.

PHILIPS David, FFA Delayed? The SNP's Plans for Further Devolution to Scotland, Londres, Institute for Fiscal Studies, 21 avril 2015, pp. 1-7.

RIDDoch Lesley, Wee White Blossom. What Post-Referendum Scotland Needs to Flourish, Édimbourg, Luath Press, 2015.

SCOTTISH CONSERVATIVES, Commission on the Future Governance of Scotland Report, Édimbourg, mai 2014.

SCOTTISH GOVERNMENT (SG), The Government Economic Strategy, Édimbourg, 2007.

SG, Fiscal Autonomy in Scotland. The Case for Change and Options for Reform, Édimbourg, février 2009.

SG, Building Security and Creating Opportunity: Economic Policy Choices in an Independent Scotland, Édimbourg, novembre 2013.

SG, Government Expenditure and Revenue Scotland 2012-2013, Édimbourg, mars 2014.

SG, Outlook for Scotland's Public Finances and the Opportunities of Independence, Édimbourg, mai 2014. SG, Scotland's Economic Strategy, Édimbourg, mars 2015. 
SG, Government Expenditure and Revenue Scotland 2013-2014, Édimbourg, mars 2015.

SG, Further Devolution beyond the Smith Commission, Édimbourg, juin 2015.

SG, Oil and Gas. Analytical Bulletin, Édimbourg, juin 2015.

SCOTTISH GREEN PARTY, Green Yes, Édimbourg, 2013.

SCOTTISH LABOUR PARTY (SLP), Powers for a Purpose. Strengthening Accountability and Empowering People, Glasgow, mars 2014.

SLP, Scottish Labour Manifesto, Glasgow, mars 2015.

SCOTTISH LIBERAL DEMOCRATS, Federalism: the Best Future for Scotland, Édimbourg, octobre 2012.

SNP, Recovery in Scotland. Make it Happen Now!, Édimbourg, 1992.

SNP, Stronger for Scotland, Édimbourg, mars 2015.

SCOTTISH PARLIAMENT (SP), Official Report, 6 février 2008, col. 5859-5904.

SP, Official Report, 28 février 2008, col. 6482-6530.

SP, Official Report, 24 septembre 2008, col. 11084-11135.

SP, Official Report, 30 octobre 2008, col. 11875-1190

SP, Official Report, 4 février 2009, col. 14649-14707.

SP, Official Report, $1^{\mathrm{er}}$ avril 2009, col. 16355-16369.

SP, Official Report, 13 mai 2009, col. 17400-17416.

SP, Official Report, 6 février 2010, col. 23364-23425.

SP, Official Report, 18 novembre 2010, col. 30578-30624.

SP, Official Report, 18 avril 2012, col. 8074-8137.

SP, Official Report, 9 octobre 2014, col. 38-59.

SP, Official Report, 18 décembre 2014, col. 38-78.

SP, Official Report, 14 janvier 2015, col. 18-70.

SP, Official Report, 4 février 2015, col. 18-69.

SP, Official Report, 19 février 2015, col. 83-103.

SP, Official Report, 10 mars 2015, col. 7-66.

SP, Official Report, 26 mars 2015, col. 53-94.

SP, Official Report, $1^{\text {er }}$ avril 2015, col. 18-67.

SP, Official Report, 30 avril 2015, col. 23-42.

SP, Official Report, 3 juin 2015, col. 20-44.

SCOTTISH PARLIAMENT, DEVOLUTION COMMITTEE, New Powers for Scotland: An Interim Report on the Smith Commission and the UK Government's Proposals, Édimbourg, SP paper 720, 14 mai 2015.

SCOTTISH SOCIALIST PARTY, Our Policies, Glasgow, 2014.

SCOTTISH TRADES UNION CONGRESS (STUC), A Just Scotland, Glasgow, février 2014.

STUC, Submission to the Smith Commission, Glasgow, octobre 2014. 
SMITH COMMISSION, Report of the Smith Commission for Further Devolution of Powers to the Scottish Parliament, Édimbourg, 27 novembre 2014.

TORRANCE David, StURGEON Nicola, A Political Life, Édimbourg, Birlinn, 2015.

\section{NOTES}

1. Cela correspond à l'examen du Scotland Bill en commission à la Chambre des communes.

2. SP, Official Report, 30 octobre 2008, col. 11882.

3. SP, Official Report, $1^{\mathrm{er}}$ avril 2009, col. 13658. L'établissement, qui avait pratiqué des investissements risqués, subissait aussi la concurrence des banques de détail, devenue plus agressive depuis l'éclatement de la crise financière.

4. SP, Official Report, 13 mai 2009, col. 17415.

5. SP, Official Report, 24 septembre 2008, col. 11122.

6. SP, Official Report, 28 février 2008, col. 6487 et 18 novembre 2010, col. 585.

7. SP, Official Report, 6 février 2008, col. 5864, 4 février 2009, col. 14649, 6 février 2010, col. 23408.

8. SP, Official Report, 18 avril 2012, col. 8074, ; HC, Hansard, 26 avril 2012, col. 1140.

9. Il avait détaillé les diverses options de réforme. SG, Fiscal Autonomy in Scotland, Édimbourg, février 2009, p. 6.

10. Le groupe de travail a fait figurer ses principes dans le titre de son second rapport («équité, personnalisation et simplicité »), précisant que la protection sociale représentait, entre 2008 et 2013, une part plus faible du PIB en Écosse que dans le reste du Royaume-Uni, EXPERT GROUP ON WELFARE, Rethinking Welfare: Fair, Personal and Simple, Édimbourg Scottish Government, mai 2014, pp. 30,74 .

11. Le rapport d'experts prônait un système fiscal stimulant la compétitivité tout en réduisant les inégalités sociales. FISCAL COMMISSION WORKING GROUP, Principles for a Modern and Efficient Tax System in an Independent Scotland, Édimbourg, Scottish Government, novembre 2013, p. 103.

12. SG, Government Expenditure and Revenue Scotland 2012-2013, Édimbourg, mars 2014, p. 5.

13. SG, Building Security and Creating Opportunity, Édimbourg, novembre 2013, chapitre 2.

14. Au printemps 2014, les taux d'activité s'élevaient à $73,3 \%$ en Écosse et $73,1 \%$ dans l'ensemble du Royaume-Uni. Quant aux coefficients de Gini, ils étaient en 2010 de 0,3 et 0,34 respectivement. 15. La production économique des petits États européens avait été plus sensible à la crise financière que celle de l'Écosse. HMG, Scotland Analysis: Assessment of a Sterling Currency Union, Londres, Stationery Office, février 2014, pp. 17, 48.

16. HMG, Scotland Analysis: Fiscal Policy and Sustainability, Londres, Stationery Office, mai 2014, p. 59.

17. SCOTTISH CONSERVATIVES, Commission on the Future Governance of Scotland Report, Édimbourg, mai 2014 ; SLP, Powers for a Purpose, Glasgow, mars 2014, 297 p. ; SCOTTISH LIBERAL DEMOCRATS, Federalism: the Best Future for Scotland, Édimbourg, octobre 2012.

18. STUC, A Just Scotland, Glasgow, février 2014, pp. 60-61.

19. Enquête de Lord Ashcroft les 17 et 18 septembre 2014, citée par MACWHIRTER Iain, Disunited Kingdom. How Westminster Won a Referendum but Lost Scotland, Glasgow, Cargo, 2014, p. 173.

20. Enquête YouGov, entre août 2014 et mars 2015, pour la "Scottish Referendum Study" financée par l'ESRC. HENDERSON Aisa \& MITCHELl James, The Scottish Question. Six Months On, Édimbourg, University of Edinburgh, 2015, p. 8.

21. Enquête Scottish Social Attitudes, réalisée pendant l'été 2014. PATERSON Lindsay, «Utopian Pragmatism », Scottish Affairs, vol. 24, n²1, 2015, p. 43. 
22. La participation a atteint $75 \%$ à Glasgow alors qu'elle dépassait rarement $40 \%$ lors des élections de 2011 à Holyrood. Cette mobilisation était l'un des enjeux du scrutin. McLean Christopher \& Thomson Douglas, «Characterization of the Disadvantaged: Explaining Differences in Levels of Support for Independence by Income Levels, Economic Activity and Socio-Economic Status ", Scottish Affairs, vol. 23, n¹, 2014, p. 130.

23. Ce coefficient s'élève à 0,72 ; il est de 0,5 avec la proportion de personnes sans diplôme et de 0,51 avec la proportion de malades de longue durée selon nos calculs.

24. sмiтн сомmission, Report of the Smith Commission for Further Devolution of Powers to the Scottish Parliament, Édimbourg, novembre 2014.

25. SP, Official Report, 18 décembre 2014, col. 49, 27 janvier 2015, col. 10, 14.

26. SG, Government Expenditure and Revenue Scotland 2013-2014, Édimbourg, mars 2015, p. 7.

27. Ce type d'écart était également envisagé par Jim McColl, chef d'entreprise et membre du groupe de conseillers du gouvernement écossais. SP, Official Report, $1^{\mathrm{er}}$ avril 2015, col. 21, 23.

28. Ces déficits nets, incluant les dépenses d'investissement, correspondaient respectivement à 8,6\% et $4 \%$ du PIB. Les chiffres pour 2013-2014 concernaient les dépenses et les revenus courants. PHILIPS David, FFA Delayed? The SNP's Plans for Further Devolution in Scotland, Londres, Institute for Fiscal Studies, 21 avril 2015, p. 4.

29. SG, Scotland's Economic Strategy, Édimbourg, mars 2015, p. 8.

30. SNP, Stronger for Scotland, Édimbourg, mars 2015, p. 8.

31. sP, Official Report, 26 mars 2015, col. 57 ; The Herald, 24 juin 2015, p. 6.

32. SP, Official Report, 19 février 2015, col. 84.

33. Il s'appuyait sur le rapport d'un groupe de pression indépendantiste. N-56, Oil and Gas. A LongTerm Plan to Maximise Economic Impact, Édimbourg, mars 2015, p. 2.

34. SP, Official Report, 10 mars 2015, col. 14.

35. Il s'élevait alors à 0,8 , alors que celui concernant le SNP n'était pas significatif selon les calculs de l'auteur.

36. SG, Further Devolution Beyond the Smith Commission, Édimbourg, 15 juin 2015, pp. 6-16.

37. HC, Hansard, 8 juin 2015, col. 944.

38. STUC, Submission to the Smith Commission, Glasgow, octobre 2014, 2.4, 3.1. Il prône une harmonisation fiscale dans l'UE. La principale confédération patronale, CBI Scotland, dénonce le surcoût et la complexité. Submission to the Smith Commission, Glasgow, octobre 2014, p. 2.

39. Selon l'Office for Budget Responsibility, organisme indépendant créé par le gouvernement britannique, la recette annuelle la plus faible des 20 dernières années ( 2 milliards) pourrait constituer la totalité du produit des 20 prochaines années. HC, Hansard, 15 juin 2015, col. 78. Le gouvernement écossais, en dépit de prévisions revues à la baisse en juin 2015, envisageait, pour la période 2016-2020, des revenus compris entre 2,4 milliards et 10,8 milliards de livres. SG, Oil and Gas. Analytical Bulletin, juin 2015, p. 17.

40. sP, Official Report, 30 avril 2015, col. 23.

41. HC, Hansard, 30 juin 2015, col. 1423. Ils avaient des propositions semblables pour le Crown estates.

42. SP, Official Report, 4 février 2015, col. 18. Il se réfère habilement à l'un des principes énoncés en 1776 par l'économiste écossais Adam Smith, selon lequel l'impôt doit être proportionnel aux ressources du contribuable, SP, Official Report, 9 octobre 2014, col. 39.

43. SP, Official Report, 3 juin 2015, col. 37.

44. FINDLAY Neil, KANE Tommy \& LEONARD Richard, « Powers for Radical Change », in BRYAN Pauline (dir.), Scotland. Myths, Realities and Radical Future, Glasgow, Red paper collective, 2015, pp. 13-14.

45. SP, Official Report, 14 janvier 2015, col. 18.

46. $\mathrm{HC}$, Hansard, 15 juillet 2015, col. 1022. 


\section{RÉSUMÉS}

La crise financière a renforcé les revendications autonomistes en Écosse (indépendance totale ou transfert de pouvoirs au Parlement écossais), considérées comme des moyens d'échapper aux politiques britanniques d'austérité. Si le SNP a tiré avantage de cette imbrication sur le plan électoral, le gouvernement écossais va devoir répondre aux attentes de la population.

Since the outbreak of the financial crisis, constitutional demands - full independence or further devolution- have been increasingly regarded as means to avoid the British Governments' austerity policies. The SNP has taken full electoral advantage of this overlap. Yet the Scottish Government will now have to meet expectations.

INDEX

Keywords : Scotland, devolution, independence, austerity, taxes

Mots-clés : Ecosse, dévolution, indépendance, austérité, impôts

\section{AUTEUR}

\section{EDWIGE CAMP-PIETRAIN}

Université de Valenciennes 